\title{
Demonstration of a Dual Alkali Rb/Cs Atomic Fountain Clock
}

\author{
J. Guéna, P. Rosenbusch, Ph. Laurent, M. Abgrall, D. Rovera, M. Lours, G. Santarelli, M.E. Tobar, S. Bize and \\ A. Clairon
}

\begin{abstract}
We report the operation of a dual $\mathrm{Rb} / \mathrm{Cs}$ atomic fountain clock. ${ }^{133} \mathrm{Cs}$ and ${ }^{87} \mathrm{Rb}$ atoms are cooled, launched, and detected simultaneously in LNE-SYRTE's FO2 double fountain. The dual clock operation occurs with no degradation of either the stability or the accuracy. We describe the key features for achieving such a simultaneous operation. We also report on the results of the first $\mathrm{Rb} / \mathrm{Cs}$ frequency measurement campaign performed with FO2 in this dual atom clock configuration, including a new determination of the absolute ${ }^{87} \mathrm{Rb}$ hyperfine frequency.
\end{abstract}

\section{INTRODUCTION}

The development of $\mathrm{Rb}$ atomic fountains was initially motivated by the prediction that the cold collision shift in $\mathrm{Rb}$ would be much reduced compared with Cs [1]. Following 2 independent experimental verifications of this prediction [2], [3], the development of a high accuracy Rb fountain frequency standard has been pursued at SYRTE. a first application of the ${ }^{87} \mathrm{Rb}$ fountain was a measurement of $\nu_{\mathrm{Rb}}$, the ground state hyperfine frequency of rubidium, performed at SYRTE with considerably improved accuracy [4]. Several $\nu_{\mathrm{Cs}} / \nu_{\mathrm{Rb}}$ measurements followed, which all involved the $\mathrm{Rb}$ fountain with Cs reference provided by the SYRTE FO1 or the FOM mobile fountain. The $\nu_{\mathrm{Rb}}$ determination performed in 2002 was chosen as a secondary representation of the SI second by the Consultative Committee for Time and Frequency (CCTF) in 2004 [5]. The main interest of precise and repeated $\mathrm{Rb} / \mathrm{Cs}$ frequency comparisons is to provide a test of variation of fundamental constants [6][8]. For this and other tests involving the alkali hyperfine frequency ratio, probing the 2 species simultaneously in the same environment was considered as a promising approach to improve the comparison. The $\mathrm{Rb} / \mathrm{Cs}$ dual fountain, dubbed FO2, developed at SYRTE aims at pursuing this goal. Following longstanding developments of $\mathrm{Rb}$ and Cs subsystems independently [7], [8], the FO2 fountain operating with either $\mathrm{Cs}$ or $\mathrm{Rb}$ as single species at a given time proved among the most accurate fountain clocks. Here we report on the first truly simultaneous operation of FO2 as a $\mathrm{Rb} / \mathrm{Cs}$ dual clock.

Manuscript received June 3, 2009; accepted Cctober 30, 2009. This work is supported by LNE. SYRTE is Unité Mixte de recherche du CNRS (UMR 8630) and is associated with Université Pierre et Marie Curie.

J. Guéna, P. Rosenbusch, Ph. Laurent, M. Abgrall, D. Rovera, G. Santarelli, S. Bize, and A. Clairon are with Laboratoire National de Métrologie-Système de Référence Temps Espace (LNE-SYRTE), Observatoire de Paris, France (e-mail: jocelyne.guena@obspm.fr).

M. E. Tobar is with University of Western Australia, School of Physics, Crawley, Australia.

Digital Object Identifier 10.1109/TUFFC.2010.1461
The key elements and techniques for dual mode operation are described in section II. section III deals with the frequency accuracy of the dual clock. The first measurements performed with the dual clock $\mathrm{FO} 2$, and associated results, are given in section IV.

\section{DUAL FoUnTAIN APPARATUS AND TECHNIQUES}

\section{A. The Dual Atom Fountain Set-Up}

Fig. 1 shows the scheme of the $\mathrm{Rb} / \mathrm{Cs} \mathrm{FO} 2$ fountain. The 2 atomic species are captured in the same region by a $\mathrm{Rb} / \mathrm{Cs}$ dual optical molasses operating in a lin $\perp$ lin configuration. $\mathrm{The} \mathrm{Rb} / \mathrm{Cs}$ optical molasses are overlapped using dedicated dichroic collimators, the key elements for simultaneous operation. The laser beams at 780 and $852 \mathrm{~nm}$, for cooling $\mathrm{Rb}$ and $\mathrm{Cs}$, respectively, are generated on 2 separate optical benches, then sent into the collimators via optical fibers and combined on a dichroic beam splitter (Fig. 2). The resulting beam is collimated using a single achromatic lens to a diameter of about $26 \mathrm{~mm}$. The realization of these collimators [9] had to meet severe constraints to ensure capture efficiency and verticality of the launch direction for both $\mathrm{Rb}$ and Cs: alignment of $\mathrm{Rb}$ and $\mathrm{Cs}$ beams to $\sim 100 \mu \mathrm{rad}$, centering of intensity profiles to $\sim 1 \mathrm{~mm}$, in addition to being nonmagnetic and removable to fit in existing magnetic shields. The 6 dual-wavelength laser beams are aligned along the axes of a 3 -D coordinate system, where the $(1,1,1)$ direction is vertical. The optical molasses are loaded from 2D-MOT presources [10] for both $\mathrm{Rb}$ and Cs. Typical loading time is $500 \mathrm{~ms}$ for both $\mathrm{Rb}$ and $\mathrm{Cs}$, with total optical power of $\sim 100 \mathrm{~mW}$ using injection-locked laser diodes for Cs and $\sim 150 \mathrm{~mW}$ using tapered optical amplifiers for $\mathrm{Rb}$. The 2D-MOTs decrease the consumption of $\mathrm{Rb}$ and $\mathrm{Cs}$ compared with previous chirp cooled atomic beams, yet at the price of decreased atomic fluxes. These presources also reduce the background pressure in the capture zone.

Both species are launched upward at the same instant with a slightly different velocity of $4.33 \mathrm{~ms}^{-1}$ (apogee $0.96 \mathrm{~m}$ ) for $\mathrm{Cs}$ and $4.16 \mathrm{~ms}^{-1}$ (apogee $0.88 \mathrm{~m}$ ) for $\mathrm{Rb}$, then cooled to $\sim 0.9$ and $1.5 \mu \mathrm{K}$, respectively, in the $\mathrm{Cs} F=$ and $\mathrm{Rb} F=2$ hyperfine ground states. There are 2 state-selection microwave cavities (at heights 64 and $139 \mathrm{~mm}$ above capture region for $\mathrm{Cs}$ and $\mathrm{Rb}$, respectively). For Cs the $F=3, m_{F}=0$ initial clock state is populated with atom number adjusted for full or half density by adiabatic population transfer [11]. For Rb, the $F=1, m_{F}=0$ initial clock state is populated by a microwave interaction from the $F=2, m_{F}=0$ state. Push beams (Fig. 1) throw out $\mathrm{Cs} / \mathrm{Rb}$ atoms remaining in the unwanted atomic states ( $F=4$ for Cs and $F=2$ for Rb). 


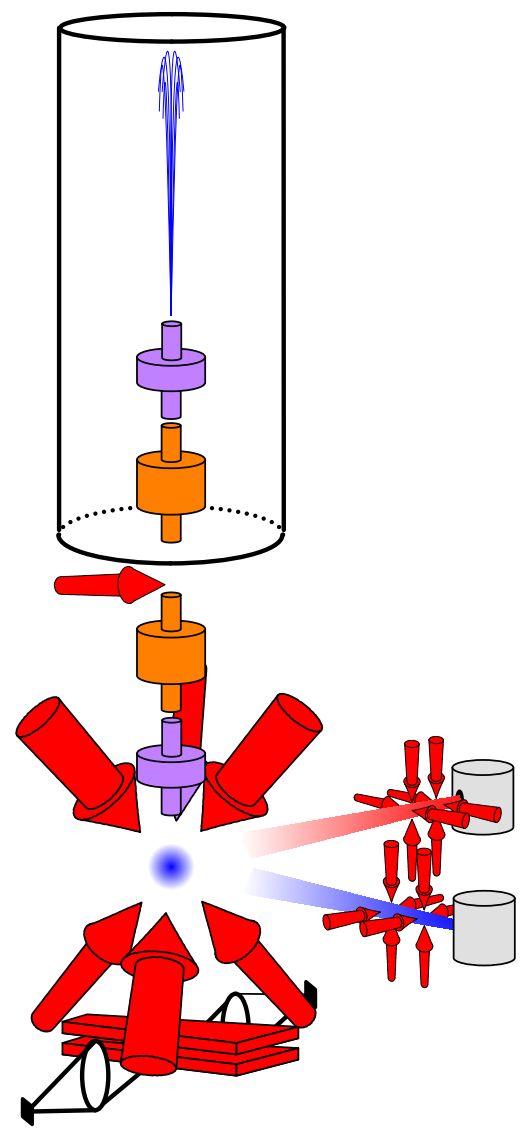

Fig. 1. Scheme of the dual fountain set-up.

The Ramsey interrogation probing the hyperfine transitions $\left({ }^{133} \mathrm{Cs} F=3 \longrightarrow F=4\right.$ at $9.192 \mathrm{GHz}$ and ${ }^{87} \mathrm{Rb}$ $F=1 \longrightarrow F=2$ at $6.834 \mathrm{GHz}$ ) consists of 2 microwave interactions $(\pi / 2$ pulses) separated by a free atomic time of flight for each species. The interactions at upward and downward passages take place in a special double microwave cavity made of 2 resonators on top of each other. The height of the resonator center above the molasses zone is $0.442 \mathrm{~m}$ for $\mathrm{Rb}$ and $0.518 \mathrm{~m}$ for $\mathrm{Cs}$. The $\mathrm{Rb} / \mathrm{Cs}$ resonators are machined out of a single copper assembly (Fig. 3) for compactness and to achieve temperature tuning for each atomic species at the same temperature. Tuning for both cavities simultaneously to within $40 \mathrm{kHz}$ of the atomic resonances occurs near $300 \mathrm{~K}$. The resonators are $T E_{011}$ cylindrical cavities with quality factors of $\sim 7100$ for $\mathrm{Cs}$ and $\sim 6000$ for Rb. Each resonator can be fed either symmetrically or asymmetrically using 2 opposite microwave feedthroughs allowing the study and reduction of first-order doppler effects related to phase gradients inside the cavity.

\section{B. Dual Detection Technique}

The detection zone is located below the molasses zone. It consists of 2 separate dual wavelength standing waves of resonant light (the second one with repumper light) allowing

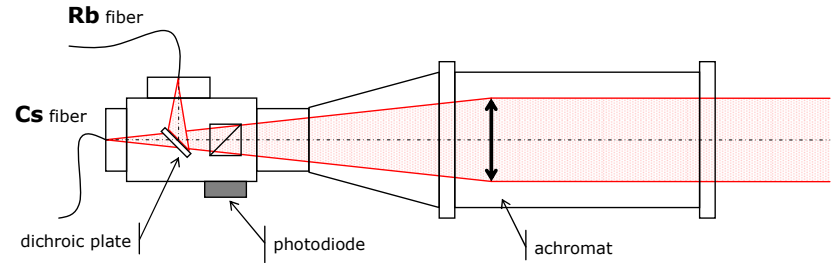

Fig. 2. Schematic of a dual collimator.

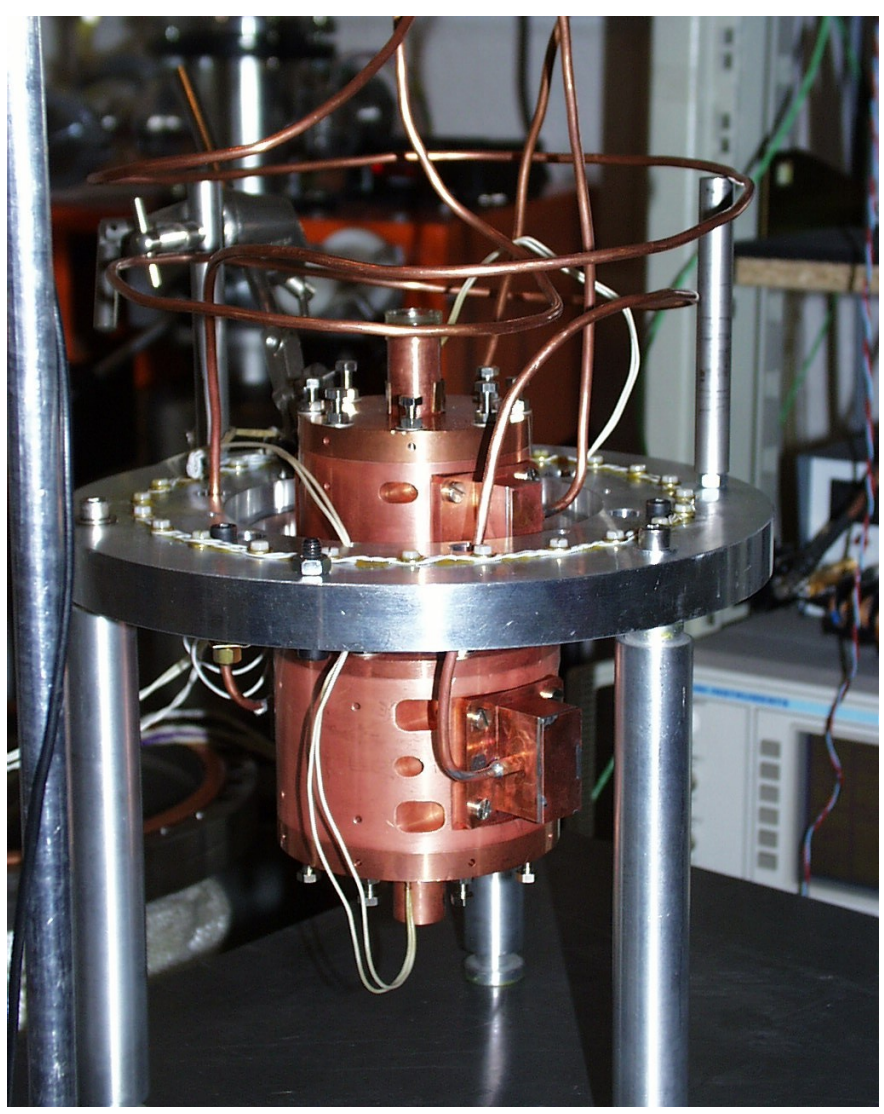

Fig. 3. The FO2 dual Rb/Cs Ramsey cavity.

detection of the 2 clock states for each atomic species by induced fluorescence. The fluorescence light is collected nonselectively onto the same photodetectors for $\mathrm{Rb}$ and $\mathrm{Cs}$ and selection between the 2 alkalis is temporal. The launch velocities are chosen to avoid collisions between the 2 atomic clouds during the entire ballistic flight (microwave state selection included), and then the launch instants are finely adjusted so that there is no overlap between the $\mathrm{Rb}$ and Cs times of flight. The difference in $\mathrm{Rb} / \mathrm{Cs}$ launch instants is of a fraction of a millisecond. a plot of the dual $\mathrm{Rb} / \mathrm{Cs}$ ballistic flight is given in Fig. 4. The typical dual clock cycle is $1.5 \mathrm{~s}$. note that the Ramsey interrogation times, and hence the Ramsey fringe widths, are equal for $\mathrm{Rb}$ and $\mathrm{Cs}(T=598 \mathrm{~ms}$, Ramsey fringe $\mathrm{FWHM} \cong 0.82 \mathrm{~Hz}$ ).

The fluorescence signal from one detection channel in dual mode is shown in Fig. 5. despite the wings of the cold atom velocity distributions (especially for $\mathrm{Rb}$ ), the overlap between $\mathrm{Rb}$ and $\mathrm{Cs}$ is insignificant. We choose to apply the detection 
beams for about $100 \mathrm{~ms}$ for the 2 atomic species consecutively without any temporal overlap. In these conditions, the signalto-noise ratio, and hence the frequency stability, for each atomic clock alone is preserved. The short-term frequency instabilities, limited by atom numbers, are at present about $3 \times 10^{-14}$ at $1 \mathrm{~s}$ for Cs at high density and $5 \times 10^{-14}$ at $1 \mathrm{~s}$ for $\mathrm{Rb}$.

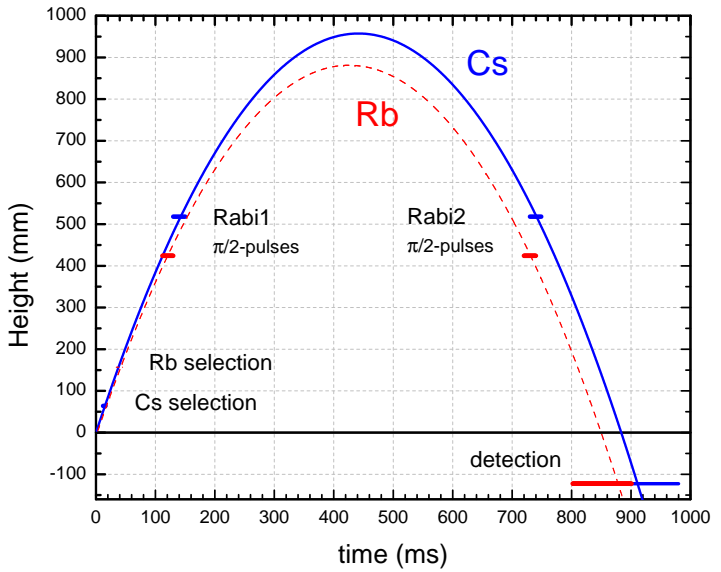

Fig. 4. $\mathrm{Rb} / \mathrm{Cs}$ ballistic flights in dual-clock configuration. Time origin is the launch instant and height origin is the molasses height.

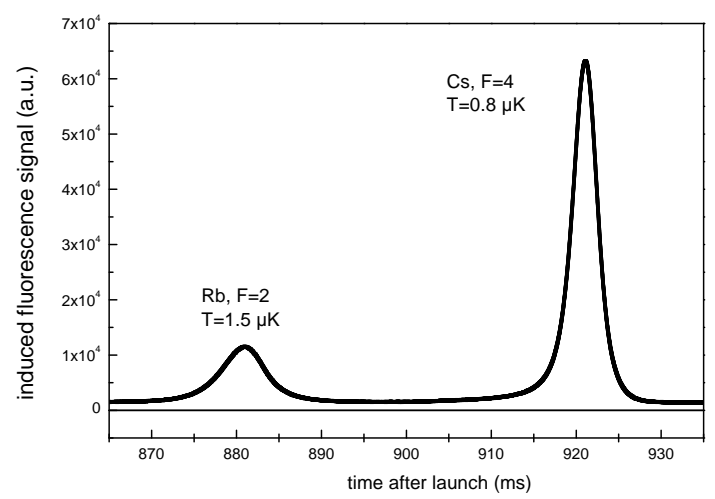

Fig. 5. Example of a dual fluorescence signal from the upper state detection channel with the time sequence given in Fig. 4. The interrogation signals for both $\mathrm{Rb}$ and $\mathrm{Cs}$ are at Ramsey center fringe resonance of the respective clock transitions, whereas for clock operation, the interrogation frequency alternates between the 2 sides of the fringe.

\section{Cs/Rb Synthesizers}

The stability requirements of the frequency synthesizers driving the clock transitions are quite stringent, and a variety of different designs have been implemented already. At LNESYRTE, the heart of the frequency synthesis is a cryogenic sapphire oscillator (CSO) operating near $11.932 \mathrm{GHz}$. a frequency offset stage already described in [12] shifts its output

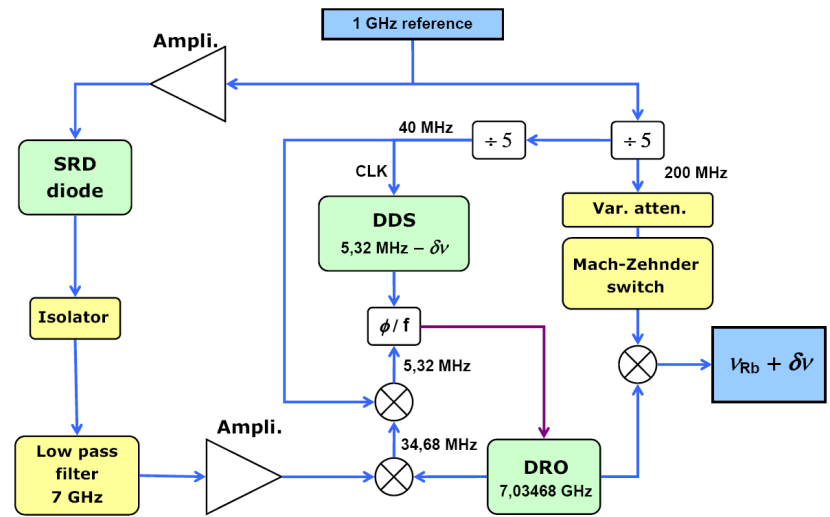

Fig. 6. Schematic of the FO2-Rb microwave synthesizer.

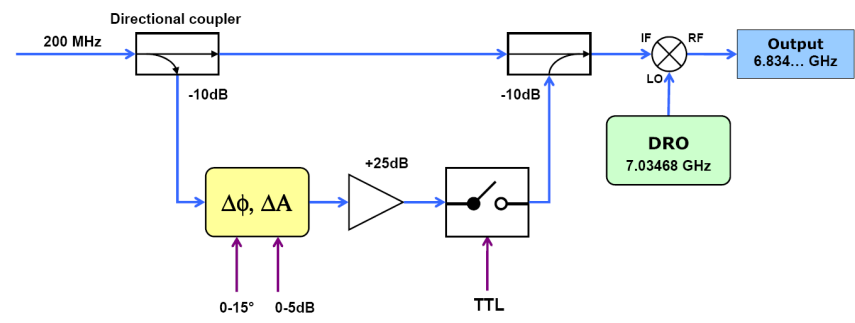

Fig. 7. The Mach-Zehnder switch in the FO2-Rb synthesizer.

signal to $11.98 \mathrm{GHz}$ with tunability using a direct digital synthesizer (DDS). The $11.98 \mathrm{GHz}$ signal reproduces the low phase noise and high short-term frequency stability of the CSO. This highly stable signal is down converted to $1 \mathrm{GHz}$ and to $100 \mathrm{MHz}$ for distribution purposes [13]. To compensate the drift of the CSO $\left(\sim 10^{-18} / \mathrm{s}\right.$ in relative frequency), the signal at $11.98 \mathrm{GHz}$ is phase-locked to the $100 \mathrm{MHz}$ output signal of a H-maser (with time constant of $1000 \mathrm{~s}$ ).

In the present configuration, the Cs synthesizer uses the low phase noise $11.98 \mathrm{GHz}$ signal to generate $9.192 \mathrm{GHz}$ in a home-built frequency chain, whereas the $1 \mathrm{GHz}$ signal is up-converted to $6.384 \mathrm{GHz}$ for $\mathrm{Rb}$ as shown in Fig. 6 . Each frequency synthesizer incorporates a computer-controlled DDS digital synthesizer with microhertz resolution to tune the interrogation signal to the clock transition. The frequency corrections applied to the DDSs are the basis for evaluating the frequency stability and frequency shifts. Each synthesizer includes a Mach-Zehnder interferometer RF switch at $400 \mathrm{MHz}$ or $200 \mathrm{MHz}$ (Fig. 7). The switch provides an extinction ratio of 60 to $70 \mathrm{~dB}$ of the microwave signal without introducing significant phase transients [14], [15]. during the clock operation, the switch turns off the microwave signal when atoms are outside the microwave cavities with the purpose of suppressing possible microwave leakage during the Ramsey inter-rogation. The phase noise power spectral density and the long-term stability of the synthesis have been extensively tested. The noise is negligible at the $10^{-14} \tau^{-1 / 2}$ level. Each synthesizer has 2 outputs with power and phase adjustments on one channel to provide symmetrical feeding of the Ramsey resonators. 


\begin{tabular}{lccc}
\hline & FO2-Cs & FO2-Rb & FO1-Cs \\
& corr. \pm unc. $\left(\times 10^{-16}\right)$ & corr. \pm unc. $\left(\times 10^{-16}\right)$ & corr. \pm unc. $\left(\times 10^{-16}\right)$ \\
\hline Quadratic Zeeman effect & $-1914.3 \pm 0.3$ & $-3468 \pm 0.7$ & $-1276.7 \pm 0.2$ \\
Blackbody radiation & $167.2 \pm 0.6$ & $120.6 \pm 1.6$ & $165.2 \pm 0.6$ \\
Cold collisions and cavity pulling & $246 \pm 2.5$ & $8.4 \pm 2.6$ & $81 \pm 2.2$ \\
First-order Doppler effect & $0 \pm 3$ & $0 \pm 2.5$ & $0 \pm 3.2$ \\
Microwave leakage \& spectral purity & $0 \pm 0.5$ & $0 \pm 0.5$ & $0 \pm 1$ \\
Others (quantum motion, & & & $0 \pm 1.7$ \\
background gas collisions, & $0 \pm 2.0$ & $0 \pm 2.0$ & 4.4 \\
Ramsey \& Rabi pulling, etc.) & 4.4 & 4.5 & \\
Total uncertainty & & & \\
\hline
\end{tabular}

TABLE I

ACCURACY BUDGET FOR SYRTE FO2-CS/RB AND FO1 FOUNTAINS.

\section{Systematics/ACCURACY Budgets}

Table I gives the budget of systematic frequency shifts and associated uncertainties for $\mathrm{FO} 2-\mathrm{Cs} / \mathrm{FO} 2-\mathrm{Rb}$ in dual clock operation. By far the largest systematic effect is the quadratic Zeeman shift and $\mathrm{Rb}$ is twice as sensitive as Cs. Great care has to be taken to measure the magnetic field (C-field) value. For this purpose we use Ramsey spectroscopy on the first-order Zeeman sensitive transitions for both $\mathrm{Cs}$ and Rb. Fig. 8 shows the $\mathrm{C}$-field maps obtained with $\mathrm{Rb}$ and $\mathrm{Cs}$ consecutively. Note that each point in this figure is the average magnetic field over the atom trajectory at different launch heights. Because the $\mathrm{Rb} / \mathrm{Cs}$ microwave cavities are not at the same height (height difference is $76 \mathrm{~mm}$ ) the 2 maps are not perfectly identical. The C-field of about $2 \mathrm{mG}$ is homogeneous at the low $10^{-3}$ level, leading to a relative frequency uncertainty less than $10^{-18}$ during clock operation at standard apogees (of $0.88 \mathrm{~m}$ for $\mathrm{Rb}$ and $0.96 \mathrm{~m}$ for $\mathrm{Cs}$ ) we periodically check the stability of the C-field. The quoted uncertainty in Table I accounts for temporal fluctuations and statistical uncertainty in the field measurement, as well as uncertainties in the Zeeman coefficients involved.

The uncertainty in the blackbody radiation frequency shift (BBR) is larger for $\mathrm{Rb}$ compared with Cs because 1) the theoretical estimate has not been carried out with as much precision as for Cs [16], and 2) no measurement of the $\mathrm{Rb}$ stark coefficient has been performed with similar accuracy as for Cs [17]. The cold collisions shift is extrapolated in real time for Cs exploiting interleaved measurements at high/low Cs density using adiabatic passage [11]. The technique is not implemented for $\mathrm{Rb}$ because the related effect is much smaller (by a factor of about 30 ) with the present atom number. Instead, we perform clock measurements alternating between 2 loading times (600 and $300 \mathrm{~ms}$ ) for $\mathrm{Rb}$. note that the atom number dependent shift includes both contributions from cold collisions and from cavity pulling due to a small cavity detuning $(\sim 250 \mathrm{kHz})$. The associated uncertainty is mainly statistical. In the future we plan to systematically perform such interleaved measurements for $\mathrm{Rb}$, just as for Cs. This will be all the more desirable if we succeed in increasing the $\mathrm{Rb}$ atom number (by increasing optical power) to improve the $\mathrm{Rb}$ clock stability. at present, the limiting systematic uncertainty is the first-order Doppler effect associated with cavity phase variations. Adjustment of the symmetric feeding of the cavities and of the launch direction provides effective reduction of the odd terms in the phase distribution. The quoted uncertainty in Table I is based on theoretical estimates of even terms of distributed cavity phase (DCP) [18], [19]. Work is ongoing on both experimental and theoretical sides to reduce the DCP un-certainty toward the $10^{-16}$ level.

An extended search for microwave leaks in the FO2-Rb/Cs fountain has been conducted using the microwave switch [14], [15]. Differential measurements with continuous or pulsed application were performed at different microwave powers. no frequency difference at the $10^{-16}$ level at the nominal power of $\pi / 2$ pulses could be concluded. The leakage effect is much smaller during regular operation with microwave switched off (extinction ratio $>50$ to $60 \mathrm{~dB}$ ) during the free time of flight.

In Table I, the overall accuracy is the quadratic sum of all systematic uncertainties. The budget for the SYRTE FO1Cs fountain is also given. This fountain was involved in the measurements presented in next section.

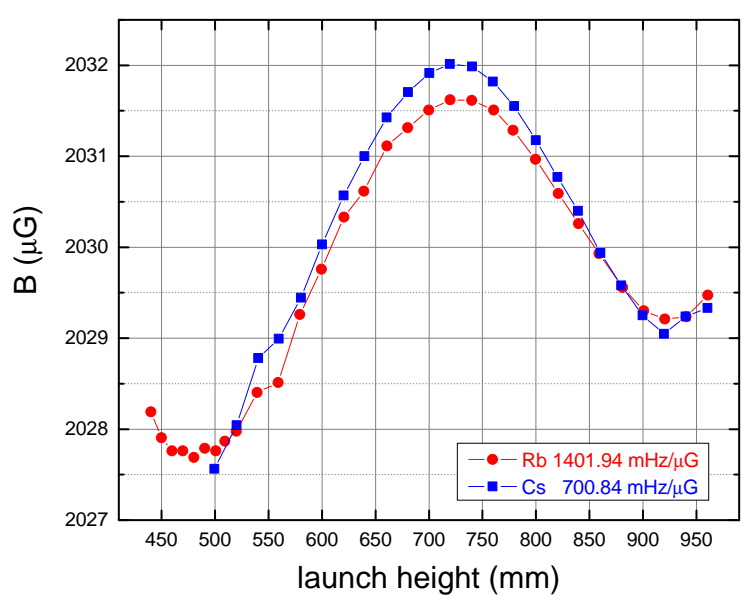

Fig. 8. C-field maps in the interrogation region probed by the FO2-Rb and FO2-Cs atoms on the Zeeman 11 transitions. 


\section{First Rb/Cs Frequency Measurements With THE DUAL ATOM CLOCK FO2}

\section{A. Rb-Cs Differential Frequency Fluctuations}

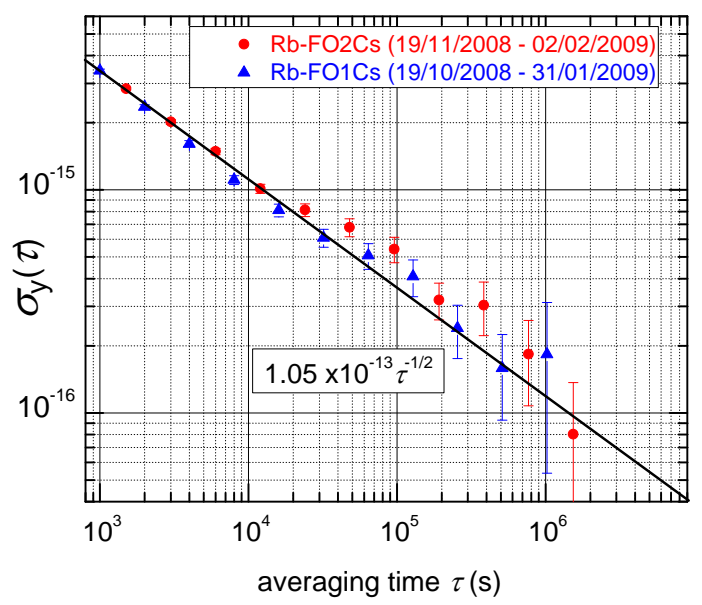

Fig. 9. Allan standard deviation for the $\mathrm{Rb} / \mathrm{Cs}$ relative frequency differences measured with FO2 as dual alkali clock. Circles: FO2-Rb/FO2-Cs comparison, triangles: FO2-Rb/FO1-Cs comparison.

The first measurement campaign with $\mathrm{FO} 2$ operating as a dual alkali atom clock took place from November 19, 2008 to January 31, 2009, covering 48 effective days of data. Fig. 9 presents the allan standard deviation for the relative frequency difference between FO2-Cs and FO2-Rb (full squares). Data are corrected for all systematic effects except for the shift dependent on $\mathrm{Rb}$ atom number which is corrected in post data processing (see section III). assuming white noise, the statistical resolution of about $10^{-16}$ is reached in 20 days.

During the same period, FO1-Cs fountain was also running. The full circles in Fig. 4 represent the allan deviation for the FO2- Rb/FO1 frequency comparison over synchronous data (36 effective days of data). It turns out that both comparisons have rather similar noise behavior. Both indicate a good rejection of the local oscillator noise: indeed, the relative frequency drift of the H-maser (of typically a few times $10^{-16}$ per day) which is conspicuous for a single clock is clearly absent in the comparison for either pair of clocks.

\section{B. New ${ }^{87}$ Rb Hyperfine Frequency Determination}

The present $\mathrm{Rb}-\mathrm{Cs}$ double campaign provides a new value for the ${ }^{87} \mathrm{Rb}$ hyperfine frequency. We take as reference the value recommended by the CCTF in 2004 [5], which is the value measured at LNE-SYRTE in 2002. Then, from the FO2$\mathrm{Rb} / \mathrm{FO} 2-\mathrm{Cs}$ comparison, we obtain the mean $\mathrm{Rb}$-Cs hyperfine relative frequency difference:

$$
\begin{aligned}
& \nu(\mathrm{FO} 2-\mathrm{Rb})(2009) / \nu_{\mathrm{Rb}}(\mathrm{CCTF})-1 \\
& \quad=(-1.68 \pm 0.65) \times 10^{-15},
\end{aligned}
$$

and from the $\mathrm{FO} 2-\mathrm{Rb} / \mathrm{FO} 1-\mathrm{Cs}$ comparison,

$$
\begin{aligned}
& \nu(\mathrm{FO} 2-\mathrm{Rb})(2009) / \nu_{\mathrm{Rb}}(\mathrm{CCTF})-1 \\
& \quad=(-1.23 \pm 0.65) \times 10^{-15} .
\end{aligned}
$$

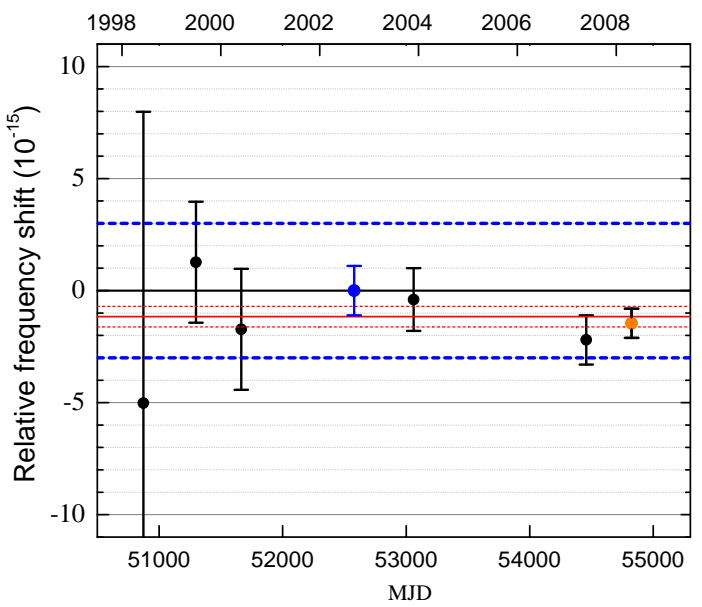

Fig. 10. Relative hyperfine frequency of ${ }^{87} \mathrm{Rb}$ relative to that of ${ }^{133} \mathrm{Cs}$ as a function of time. The data point at MJD 52579 chosen as the frequency reference is the value recommended by CCTF 2004. The thick dotted blue lines correspond to the recommended uncertainty. The red continuous line having an offset is the result of a weighted least square fit to all the data by a constant value. The thin dotted red lines correspond to the fit uncertainty at $1 \sigma$.

The 2 results are consistent within the error bars, which are dominated by the systematic uncertainties (Table I). We choose to average them with equal weights. This yields the absolute ${ }^{87} \mathrm{Rb}$ hyperfine frequency

$$
\nu(\mathrm{FO} 2-\mathrm{Rb})(2009)=6834682610.904314(4) \mathrm{Hz} .
$$

The recommended value

$$
\nu_{\mathrm{Rb}}(\mathrm{CCTF}-2004)=6834682610.904324(21) \mathrm{Hz}
$$

within the assigned relative uncertainty of $3 \times 10^{-15}$ is consistent with the new determination.

\section{Overview of Rb-Cs Frequency Comparisons}

Fig. 10 shows the results of the Rb-Cs frequency measurements performed at LNE-SYRTE since 1998. For the first 6 points, FO2-Rb was operating as a single clock and the Cs reference was FO1, the FOM mobile fountain, or both. The present result with FO2 as dual $\mathrm{Rb} / \mathrm{Cs}$ clock (last point) is perfectly consistent with previous results. A weighted least squares fit to the data by a constant has a $\chi^{2}$-goodness of fit probability of $Q=0.75$, indicating internal consistency of the data (weighted mean $-1.16 \times 10^{-15}$, uncertainty $0.46 \times 10^{-15}$ at $1 \sigma)$.

\section{Summary AND OUTLOOKS}

In this paper, we have presented the first demonstration of a dual $\mathrm{Rb} / \mathrm{Cs}$ alkali clock with the 2 species simultaneously probed in the same device. As it is implemented, the simultaneous clock operation preserves the stability and accuracy of each clock operated alone. It also preserves the possibility of configuring the 2 clocks independently for a large variety of measurements. 
To demonstrate the capability of this setup, we have used the dual fountain to perform a new absolute frequency measurement of the $\mathrm{Rb}$ hyperfine frequency. This measurement has a slightly improved uncertainty and is consistent with all measurements performed before the implementation of the simultaneous operation with $\mathrm{Rb}$ and $\mathrm{Cs}$.

For long-term operation, for $\mathrm{Rb} / \mathrm{Cs}$ comparisons over extended period of time and their application to testing the stability of fundamental constants, the dual fountain setup provides a practical gain of being a single device in a single room with a single operator. Moreover, the collocated interrogation of both species allows a partial cancelation of some systematic shifts in the measurement of the $\mathrm{Rb} / \mathrm{Cs}$ frequency ratio. For instance, in fractional terms, the $\mathrm{Rb} / \mathrm{Cs}$ ratio is $\sim 2.6$ times less sensitive to the blackbody radiation shift than $\mathrm{Rb}$ or Cs alone. It is $\sim 2$ times less sensitive to the secondorder Zeeman shift than $\mathrm{Rb}$ alone. Also, temporally matched interrogation of both atoms allows common-mode rejection of the interrogation oscillator phase noise. This can lead to large improvement when the stability is limited by the oscillator noise, as shown in [20]. However, this advantage no longer exists when quantum noise limited stability is achieved by using an ultra low noise interrogation signal as delivered by a cryogenic sapphire oscillator [21] or a femtosecond laser [22].

The dual-fountain configuration also opens the way to several new measurements. For instance, a modified version of the lorentz invariance test performed with FO2-Cs [23] could be performed using $\mathrm{Rb}$ and $\mathrm{Cs}$ simultaneously. The use of $\mathrm{Rb}$ together with Cs would extend the test to a different sector of the theoretical framework. Furthermore, magnetic field fluctuations which were limiting the stability of the measurements reported in [23] would be strongly eliminated by commonmode rejection. The dual fountain configuration also opens the possibility of studying $\mathrm{Rb} / \mathrm{Cs}$ cold collisions. For time and frequency metrology applications, the time sequence is carefully chosen to avoid $\mathrm{Rb} / \mathrm{Cs}$ collisions. However, the FO2 fountain set-up offers flexibility in the $\mathrm{Rb} / \mathrm{Cs}$ time sequences. Parameters can be easily adjusted to finely tune collision energy between the 2 clouds.

\section{REFERENCES}

[1] B. Kokkelmans, B. Verhaar, K. Gibble, and D. Heinzen, "Predictions for laser cooled Rb clocks," Phys. Rev. A., vol. 56, p. R4389, 1997.

[2] C. Fertig and K. Gibble, "Measurement and cancellation of the cold collision frequency shift in an ${ }^{87} \mathrm{Rb}$ fountain clock," Phys. Rev. Lett., vol. 85, p. 1622, 2000.

[3] Y. Sortais, S. Bize, C. Nicolas, A. Clairon, C. Salomon, and C. Williams, "Cold collision frequency shifts in a ${ }^{87} \mathrm{Rb}$ fountain," Phys. Rev. Lett., vol. 85 , p. $3117,2000$.

[4] S. Bize, Y. Sortais, M. Santos, C. Mandache, A. Clairon, and C. Salomon, "High-accuracy measurement of the ${ }^{87} \mathrm{Rb}$ ground-state hyperfine splitting in an atomic fountain," Europhys. Lett., vol. 45, no. 5, p. 558, 1999.

[5] BIPM-CCTF 2004, "Recommendation CCTF-1 2004 concerning secondary representations of the second", p. 38 (2004).

[6] H. Marion, F. Pereira Dos Santos, M. Abgrall, S. Zhang, Y. Sortais, S. Bize, I. Maksimovic, D. Calonico, J. Grünert, C. Mandache, P. Lemonde, G. Santarelli, P. Laurent, A. Clairon, and C. Salomon, "Search for variations of fundamental constants using atomic fountain clocks," Phys. Rev. Lett., vol. 90, p. 150801, 2003.
[7] S. Bize, P. Laurent, M. Abgrall, H. Marion, I. Maksimovic, L. Cacciapuoti, J. Grünert, C. Vian, F. Pereira dos Santos, P. Rosenbusch, P. Lemonde, G. Santarelli, P. Wolf, A. Clairon, A. Luiten, M. Tobar, and C. Salomon, "Advances in ${ }^{133}$ Cs fountains," C. R. Physique, vol. 5, p. 829, 2004

[8] - "Cold atom clocks and applications," J. Phys. B: Atomic, Molecular and Optical Physics, vol. 38, no. 9, pp. S449-S468, 2005.

[9] F. Chapelet, "Fontaine atomique double de Césium et de Rubidium avec une exactitude de quelques $10^{-16}$ et applications," Ph.D. dissertation, Université de Paris XI, 2008

[10] K. Dieckmann, R. J. C. Spreeuw, M. Weidemüller, and J. T. M. Walraven, "Two-dimensional magneto-optical trap as a source of slow atoms," Phys. Rev. A, vol. 58, no. 5, p. 3891, Nov 1998.

[11] F. Pereira Dos Santos, H. Marion, S. Bize, Y. Sortais, A. Clairon, and C. Salomon, "Controlling the cold collision shift in high precision atomic interferometry," Phys. Rev. Lett., vol. 89, p. 233004, 2002.

[12] D. Chambon, S. Bize, M. Lours, F. Narbonneau, H. Marion, A. Clairon, G. Santarelli, A. Luiten, and M. Tobar, "Design and realization of a flywheel oscillator for advanced time and frequency metrology," Rev. Sci. Instrum., vol. 76, p. 094704, 2005.

[13] D. Chambon, M. Lours, F. Chapelet, S. Bize, M. E. Tobar, A. Clairon, and G. Santarelli, "Design and metrological features of microwave synthesizers for atomic fountain frequency standard," IEEE Trans. Ultr Ferr. Freq. Contr., vol. 54, p. 729, 2007.

[14] G. Santarelli, G. Governatori, D. Chambon, M. Lours, P. Rosenbusch, J. Guéna, F. Chapelet, S. Bize, M. Tobar, P. Laurent, T. Potier, and A. Clairon, "Switching atomic fountain clock microwave interrogation signal and high-resolution phase measurements," Ultrasonics, Ferroelectrics and Frequency Control, IEEE Transactions on, vol. 56, no. 7, pp. 1319-1326, July 2009.

[15] J. Guéna, F. Chapelet, P. Rosenbusch, P. Laurent, M. Abgrall, G. Rovera, G. Santarelli, S. Bize, A. Clairon, and M. Tobar, "New measurement of the rubidium hyperfine frequency using LNE-SYRTE fountain ensemble," in Frequency Control Symposium, 2008 IEEE International, may 2008, pp. $366-370$.

[16] E. J. Angstmann, V. A. Dzuba, and V. V. Flambaum, "Frequency shift of hyperfine transitions due to blackbody radiation," Phys. Rev. A, vol. 74 , p. $023405,2006$.

[17] E. Simon, P. Laurent, and A. Clairon, "Measurement of the Stark shift of the Cs hyperfine splitting in an atomic fountain," Phys. Rev. A., vol. 57, p. 436, 1998.

[18] R. Li and K. Gibble, "Distributed cavity phase and the associated power dependence," Frequency Control Symposium and Exposition, 2005. Proceedings of the 2005 IEEE International, pp. 99-104, Aug. 2005.

[19] R. Schröder, U. Hübner, and D. Griebsch, "Design and realization of the microwave cavity in the ptb caesium atomic fountain clock csf1," Ultrasonics, Ferroelectrics and Frequency Control, IEEE Transactions on, vol. 49, no. 3, pp. 383-392, March 2002.

[20] S. Bize, Y. Sortais, P. Lemonde, S. Zhang, P. Laurent, G. Santarelli, C. Salomon, and A. Clairon, "Interrogation oscillator noise rejection in the comparison of atomic fountains," Ultrasonics, Ferroelectrics and Frequency Control, IEEE Transactions on, vol. 47, no. 5, pp. 1253-1255, Sep 2000.

[21] G. Santarelli, P. Laurent, P. Lemonde, A. Clairon, A. G. Mann, S. Chang, A. N. Luiten, and C. Salomon, "Quantum projection noise in an atomic fountain: A high stability cesium frequency standard," Phys. Rev. Lett., vol. 82, no. 23, p. 4619, 1999.

[22] J. Millo, M. Abgrall, M. Lours, E. M. L. English, H. Jiang, J. Guéna, A. Clairon, M. E. Tobar, S. Bize, Y. L. Coq, and G. Santarelli, "Ultralow noise microwave generation with fiber-based optical frequency comb and application to atomic fountain clock," Applied Physics Letters, vol. 94, no. 14, p. 141105, 2009.

[23] P. Wolf, F. Chapelet, S. Bize, and A. Clairon, "Cold atom clock test of Lorentz Invariance in the matter sector," Phys. Rev. Lett., vol. 96, no. 6 , p. 060801,2006 